\title{
Synthesis, surface characterization and optical properties of 3-thiopropionic acid capped $\mathrm{ZnS}$ :Cu nanocrystals
}

\author{
ASHISH TIWARI*, S A KHAN ${ }^{\dagger}$ and R S KHER ${ }^{\dagger \dagger}$ \\ Department of Chemistry, ${ }^{\dagger}$ Department of Physics, Government E R R Science P G College, Bilaspur 495 006, India \\ ${ }^{\dagger}$ Government College Seepat, Bilaspur 495 555, India
}

MS received 28 September 2010; revised 25 January 2011

\begin{abstract}
Thiopropionic acid (TPA) capped ZnS:Cu nanocrystals have been successfully synthesized by simple aqueous method. Powder X-ray diffraction (XRD) studies revealed the particle size to be $4.2 \mathrm{~nm}$. Surface characterization of the nanocrystals by FTIR spectroscopy has been done and the structure for surface bound TPA based on spectral analysis was proposed. The optical studies were done using UV-VIS spectroscopy and particle size and diameter polydispersity index (DPI) were calculated. Photoluminescence (PL) spectrum reveals emission related to the transition from conduction band of $\mathrm{ZnS}$ to $t_{2}$ level of $\mathrm{Cu}^{2+}$. Electron microscopy was also done by scanning electron microscopy (SEM).
\end{abstract}

Keywords. ZnS:Cu nanocrystals; 3-thiopropionic acid; diameter polydispersity index; photoluminescence; powdered XRD; electron microscopy.

\section{Introduction}

Nanoscience and nanotechnology conjures up visions of making, imaging, manipulating and utilizing materials that are really small (Pradeep 2007). Semiconductor nanosized and quantum confined materials are now poised to revolutionize the electronic, chemical, biotechnology and biomedical fields (Havanscak 2003; Larson et al 2003; Daniel and Astruc 2004; Sapra et al 2005). Functionalization of surface is one of the most important aspects of nanoparticles fabrication (Jun et al 2006). Doped ZnS semiconductor materials have a wide range of applications in electroluminescence devices, phosphors, light emitting displays, and optical sensors. Accordingly, study of luminescence properties of $\mathrm{ZnS}$ has received special attention. The bulk $\mathrm{ZnS}$ peak is expected at $338 \mathrm{~nm}(3.67 \mathrm{eV})$. Many of the $\mathrm{ZnS}$ systems were made using complicated methods with organic solvents, which were not suitable for bioimaging applications (Zhang and Li 2004; Kuzuya et al 2005; Ehlert et al 2008). With the decrease of particle size extremely particle high surface to volume ratio causes the surface states to act as luminescence quenching centres. Hence, the passivation of surface is of crucial importance for the application of luminescent semiconductors (Panda and Chaudhuri 2007). Surfactants play an important role in the control of dispersed particle sizes, optical properties and suitability by passivating surface of the nanocrystal (Chan and Nie 1998). 3-Thiopropionic acid, also named as $\beta$-mercaptopropionic acid, contains two functional groups, thiol and carboxylic acid which can be

*Author for correspondence (ashisht048@gmail.com) deprotonated allowing it to bind to surface of the nanoparticles. Doped $\mathrm{ZnS}$ nanocrystals can be considered for fluorescence biolabelling agent due to its long luminescence lifetime (Suyver et al 2001) and no elemental toxicity. Here, we present the synthesis of thiopropionic acid capped $\mathrm{ZnS}: \mathrm{Cu}$ nanocrystals by simple aqueous method that avoids the detrimental organometallic precursors and high temperature. The obtained nanocrystals were characterized for their morphology and structure. The optical properties were also investigated.

\section{Experimental}

\subsection{Materials and methods}

A.R. grade zinc acetate dihydrate, copper acetate monohydrate, sodium sulphide nonahydrate, mercaptopropionic acid (TPA), ethanol etc were obtained commercially and used without further purification. Double distilled water was used for all sample preparation and dilution process. In the present procedure $3.5 \mathrm{ml}$ of 3-thiopropionic acid was dissolved in $36 \mathrm{ml}$ of $\mathrm{D} / \mathrm{D}$ water and stirred for $5 \mathrm{~min}$. While stirring continuously, $5 \mathrm{ml}$ of $1 \mathrm{M}$ zinc acetate solution as also the required amount of copper acetate solution was added drop wise to the above solution. The molar concentration of $\mathrm{Cu}^{2+}$ to $\mathrm{Zn}^{2+}$ was fixed at $0 \cdot 1 \%$. $\mathrm{pH}$ of the solution was adjusted to 12 with $2 \mathrm{~N} \mathrm{NaOH}$. The above mixture was stirred for $10 \mathrm{~min}$. Then $5 \mathrm{ml}$ of $1 \mathrm{M}$ sodium solution was added to this mixture drop wise. After $5 \mathrm{~min} 15 \mathrm{ml}$ of $1 \mathrm{M}$ zinc acetate solution was again added to the above solution. A transparent solution was 
obtained. To obtain powdered nanocrystals, cold ethanol precipitation was done, the precipitate was isolated by centrifugation, washed several times with ethanol to remove adhered impurities and finally dried in an oven at $60^{\circ} \mathrm{C}$.

The prepared sample was characterized by powder XRD using Rigaku Miniflex with $\mathrm{Cu} \mathrm{K} \alpha(1.5406 \AA)$ radiation in a $2 \theta$ range of $20^{\circ}-70^{\circ}$ at a step size of $0.02^{\circ}(2 \theta)$. The FTIR spectrum was recorded on SHIMADZU FTIR 8400S spectrophotometer. UV-VIS spectra was recorded on a Systronic UV spectrophotometer 119. The scanning electron microscopy (SEM) measurements were recorded on a FEI Quanta 200 microscope equipped with an EDX unit. The PL of the as prepared nanoparticles was recorded at room temperature using SHIMADZU RF 5301 PC Spectrofluorophotometer.

\section{Results and discussion}

\subsection{Reaction mechanism}

The following mechanism is proposed for the synthesis of TPA capped $\mathrm{ZnS}: \mathrm{Cu}$ nanocrystals.

At $\mathrm{pH} 6 \cdot 5-8$,

$$
\mathrm{Zn}^{2+}+\mathrm{OH}^{-} \rightarrow \mathrm{Zn}(\mathrm{OH})_{2} \text {. }
$$

At $\mathrm{pH}>9$,

$$
\begin{aligned}
& m \mathrm{Zn}(\mathrm{OH})_{2}+n \mathrm{TPA} \rightarrow \mathrm{Zn}_{m}(\mathrm{TPA})_{n}+m \mathrm{OH}^{-}, \\
& \mathrm{Zn}_{m}(\mathrm{TPA})_{n}+m \mathrm{~S}^{2-} \rightarrow(\mathrm{ZnS})_{m}(\mathrm{TPA})_{n} .
\end{aligned}
$$

At slightly alkaline condition, $\mathrm{Zn}^{2+}$ ion reacts with $\mathrm{OH}^{-}$ to form zinc hydroxide. Under more basic concentration, $\mathrm{Zn}(\mathrm{OH})_{2}$ forms complex through chelating interactions of carboxyl and dissociated thiol. When $\mathrm{Na}_{2} \mathrm{~S}$ is introduced, $\mathrm{S}^{2-}$ may destroy the complex and combine with $\mathrm{Zn}^{2+}$ due to strong interaction than those between $\mathrm{Zn}^{2+}$ and deprotonated TPA (Crisponi et al 2002). Residual thiopropionic chelated complex may be adsorbed on the surface of growing $\mathrm{ZnS}$ nuclei and form a denser covering layer which may block further particle growth.

The solubility of $\mathrm{CuS}$ is less than that of $\mathrm{ZnS}$ hence, it seems that $\mathrm{Zn}^{2+}$ and $\mathrm{Cu}^{2+}$ cannot be coprecipitated with $\mathrm{S}^{2-}$ ions but thiol group present in the thiopropionic acid minimizes the solubility difference between $\mathrm{CuS}$ and $\mathrm{ZnS}$ by coordinating with $\mathrm{Cu}^{2+}$ and thus the possibility of coprecipitation of $\mathrm{ZnS}$ and $\mathrm{CuS}$ can be greatly improved during the synthesis and this makes it possible to obtain $\mathrm{ZnS}: \mathrm{Cu}$ nanoparticles in which $\mathrm{Cu}^{2+}$ ions replace the $\mathrm{Zn}^{2+}$ ions in the lattice.

\subsection{Grain size studies}

Figure 1 shows the measured XRD pattern of $\mathrm{ZnS}: \mathrm{Cu}-$ TPA. Three broad peaks corresponding to the (111), (220) and (311) planes were observed. Crystallite size of capped

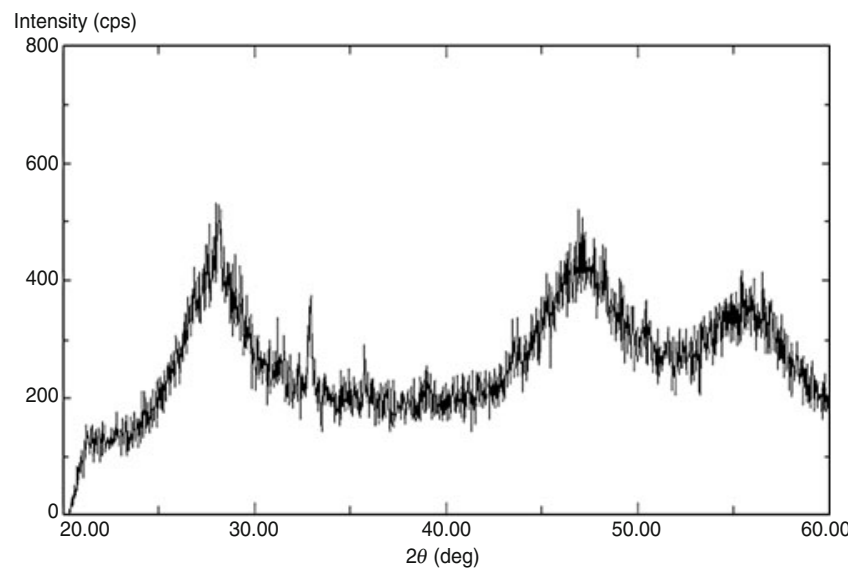

Figure 1. XRD diffraction pattern of TPA capped $\mathrm{ZnS}: \mathrm{Cu}$ nanocrystals.

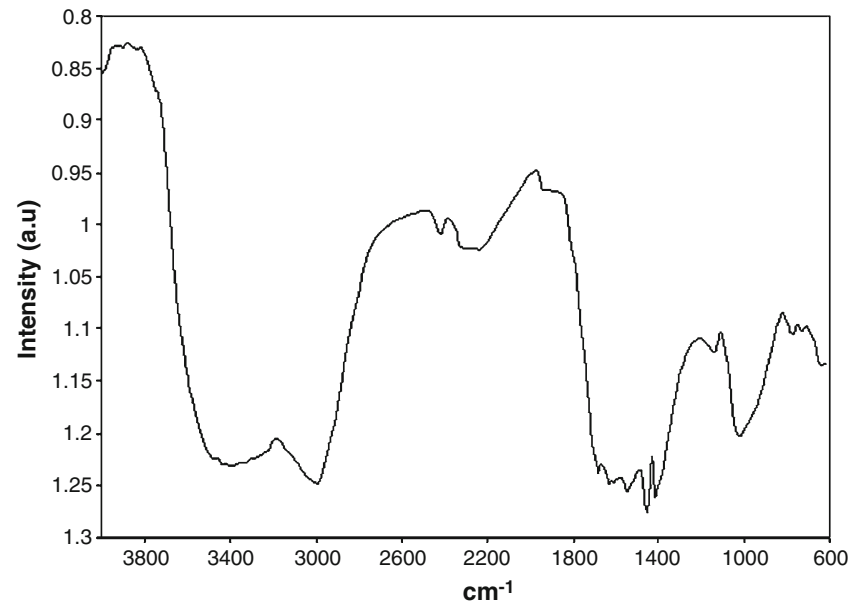

Figure 2. FTIR spectrum of TPA capped $\mathrm{ZnS}: \mathrm{Cu}$ nanocrystals.

$\mathrm{ZnS}: \mathrm{Cu}$ was calculated by following Scherrer's equation (Guinier 1963)

$$
D=K \lambda / \beta \cos \theta .
$$

The average size calculated from the above formula is $4.2 \mathrm{~nm}$. The broadening of the XRD peak indicated the formation of $\mathrm{ZnS}$ nanocrystals.

\subsection{FTIR spectra}

The form of the 3-TPA adsorbed on the $\mathrm{ZnS}: \mathrm{Cu}$ nanoparticles is inferred from FTIR spectra as shown in figure 2. The broad feature between 3000 and $3500 \mathrm{~cm}^{-1}$ is due to $\mathrm{O}-\mathrm{H}$ stretching of carboxylic group. The peak at $1414 \mathrm{~cm}^{-1}$ is due to bending vibration of methylene groups in thiopropionic acid while the peak at $2914 \mathrm{~cm}^{-1}$ is due to $\mathrm{CH}_{2}$ stretching mode of TPA. The $\mathrm{C}-\mathrm{O}$ stretching vibration is observed 
between 1016 and $1103 \mathrm{~cm}^{-1}$. Due to deprotonation of carboxylic acid group the $\mathrm{C}=\mathrm{O}$ stretching band of neat TPA is switched to an antisymmetric OCO stretch, $1630 \mathrm{~cm}^{-1}$. The $\mathrm{O}-\mathrm{H}$ bend is observed at $1451 \mathrm{~cm}^{-1}$. The absence of S-H stretch at $2554 \mathrm{~cm}^{-1}$ suggests that TPA exists as thiolate on the nanocrystals surface.

\subsection{Optical absorption studies}

Absorption spectrum of TPA capped $\mathrm{ZnS}: \mathrm{Cu}$ nanoparticles have been studied by dispersing $0.001 \mathrm{~g}$ of the sample in $1 \mathrm{ml}$ of water as shown in figure 3 . The spectrum reveals that the intensity increases toward shorter wavelength and attains a maximum at $290 \mathrm{~nm}$. It can be seen that a broad absorption peak is obtained which may be attributed to the formation of nanoaggregates of the nanoparticles as can be seen from the SEM image. The doping of $\mathrm{Cu}^{2+}$ does not change the bandgap significantly due to very little concentration since the particle size does not change appreciably but can drastically change the luminescent properties. Optical excitation of electrons across the bandgap is strongly allowed transition thus it causes an abrupt increase in the absorptivity at wavelength corresponding to gap energy. Blue shifting of absorption peak (bulk $345 \mathrm{~nm}$ ) is due to quantum confinement of the excitons present in the sample resulting in more discrete energy of the spectrum of individual nanoparticles. The

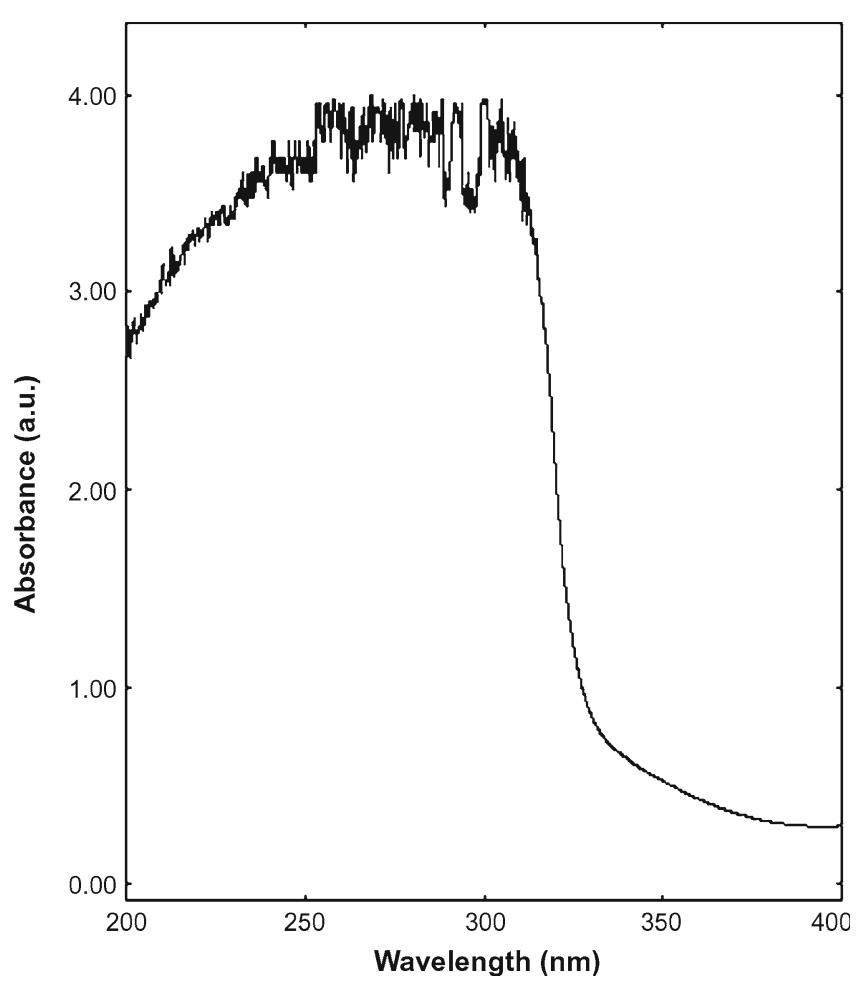

Figure 3. Absorption spectrum of TPA capped $\mathrm{ZnS}: \mathrm{Cu}$ nanocrystals. average particle size present in the nanocolloid can be determined by using the mathematical model of effective mass approximation (Brus 1986). The following equation derived, describes the particle size ( $r$, radius) as a function of peak absorbance wavelength $\left(\lambda_{\mathrm{p}}\right)$ for $\mathrm{ZnS}$ nanocrystals.

$$
\begin{aligned}
r(\mathrm{~nm})= & \left\{-0 \cdot 2963+\sqrt{\left(-40 \cdot 1970+13620 / \lambda_{\mathrm{p}}\right)}\right\} / \\
& \left\{-7 \cdot 34+2481 \cdot 6 / \lambda_{\mathrm{p}}\right\} .
\end{aligned}
$$

The particle size obtained is $3.8 \mathrm{~nm}$ for TPA capped $\mathrm{ZnS}$ nanoparticles, which is consistent with the value obtained by Scherrer's formula.

\subsection{Diameter polydispersity index (DPI)}

Width of the particle size distribution can be related to $d_{1 / 2}$ value which is given by the difference between the particle diameter calculated from the edge of the absorption and the particle diameter calculated from the position of exciton shoulder, $\lambda_{\mathrm{e}}$. Assuming a gaussian distribution it is possible to convert $d_{1 / 2}$ into a diameter polydispersity index (DPI) (Moffit et al 1995) according to the following formula

$$
\mathrm{DPI}=\left(\sigma_{\mathrm{d}} / d_{\mathrm{s}}\right)^{2}+1,
$$

where $\sigma_{\mathrm{d}}$ is the standard deviation taken as half of $d_{1 / 2}$ and $d_{\mathrm{s}}$ the mean particle diameter which corresponds to position of exciton shoulder. Taking into account that DPI $=1$ defines a monodispersed sample, the calculated value of DPI implies a relatively low size of polydispersity of $\mathrm{ZnS}$ nanoparticles. The calculation of DPI is shown in table 1 .

\subsection{Electron microscopy}

The microstructure of the nanocrystals has been examined by scanning electron microscopy (SEM) and energy dispersive $\mathrm{X}$-ray (EDX) analysis of the prepared nanocrystals is shown in figures 4 (a), (b) and (c), respectively.

\subsection{PL spectrum of TPA capped ZnS:Cu nanoparticles}

Figure 5 shows the room temperature PL excitation and emission spectra of TPA capped $\mathrm{ZnS}: \mathrm{Cu}$ nanoparticles. The excitation peak was obtained at around $240 \mathrm{~nm}$. The

Table 1. Diameter polydispersity index for TPA capped $\mathrm{ZnS}: \mathrm{Cu}$.

\begin{tabular}{cccccc}
\hline$\lambda_{\mathrm{e}}$ & $\lambda_{\mathrm{p}}$ & $d_{\mathrm{e}}$ & $d_{\mathrm{p}}$ & $d_{1 / 2}$ & DPI \\
\hline $313 \mathrm{~nm}$ & $290 \mathrm{~nm}$ & $5.1 \mathrm{~nm}$ & $3.8 \mathrm{~nm}$ & $1.3 \mathrm{~nm}$ & 1.02 \\
\hline
\end{tabular}



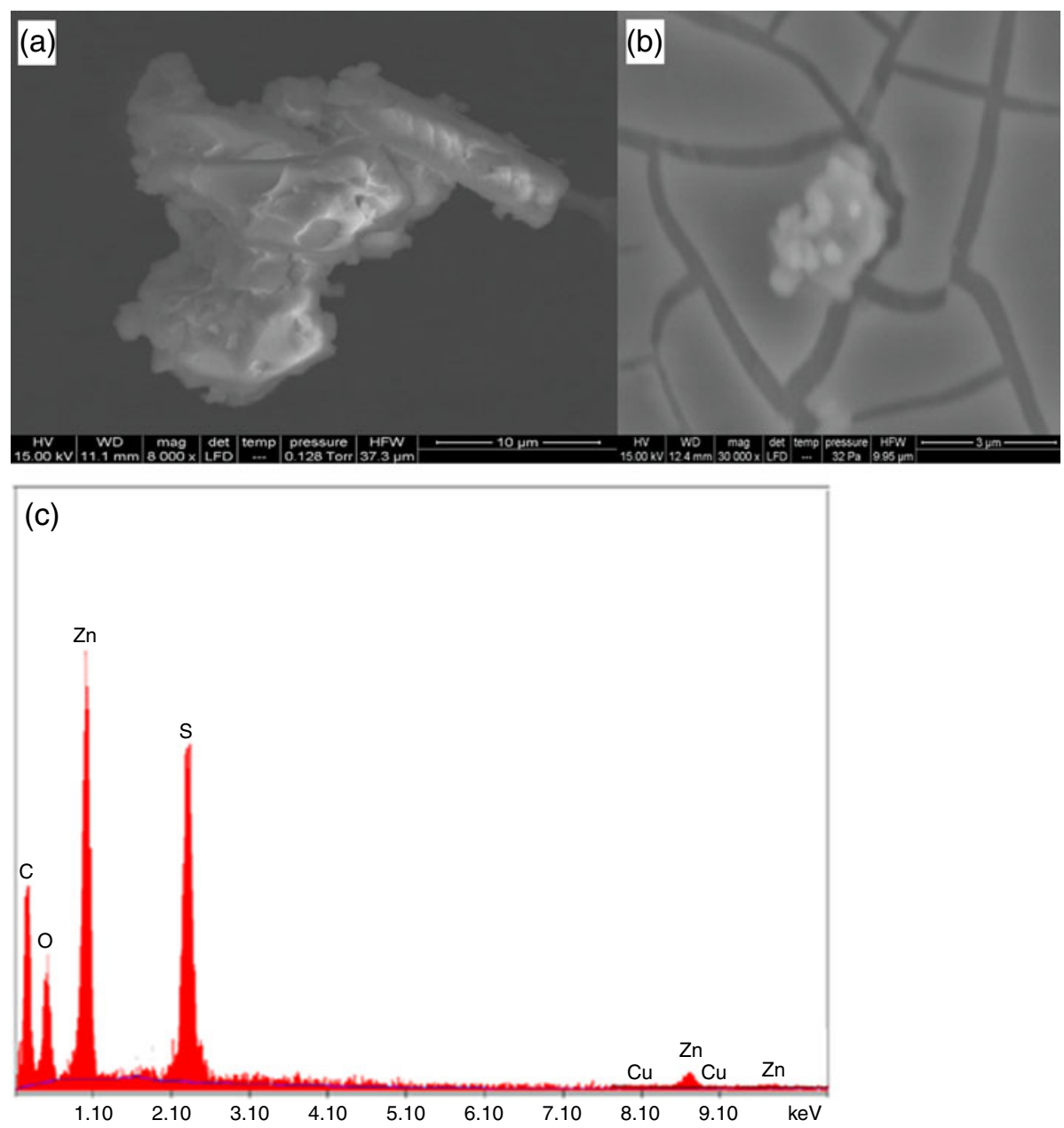

Figure 4. (a), (b) SEM images of TPA capped ZnS nanocrystals and (c) EDX spectrum of TPA capped $\mathrm{ZnS}: \mathrm{Cu}$ nanocrystals.

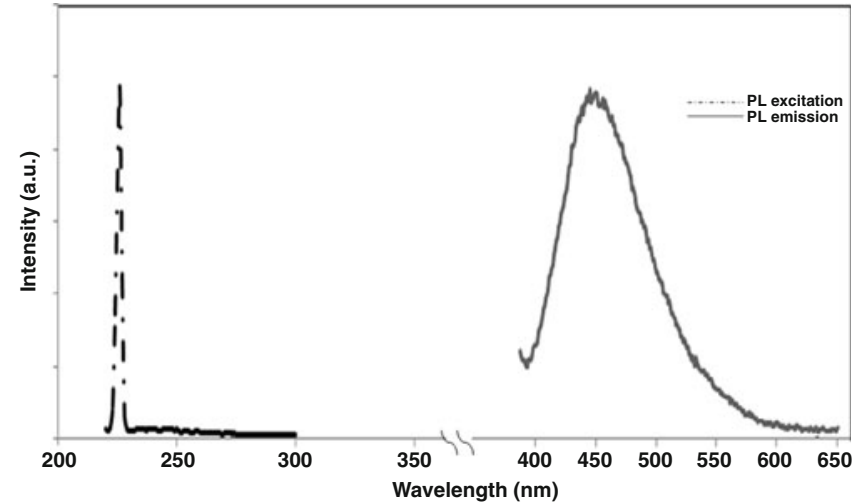

Figure 5. PL spectrum of TPA capped ZnS:Cu nanocrystals.

incorporation of $\mathrm{Cu}^{2+}$ in the $\mathrm{ZnS}$ host can be confirmed from the fact that undoped $\mathrm{ZnS}$ shows an emission at around $424 \mathrm{~nm}$ (Warad et al 2005) resulting from the transition of electrons from the shallow states near the conduction band to sulphur vacancies present near the valence band. In the present study, blue emission at about $460 \mathrm{~nm}$ is attributed to the transition between conduction band of $\mathrm{ZnS}$ host and the acceptor like $t_{2}\left(d^{9}\right)$ state of $\mathrm{Cu}$ (Manzoor et al 2004; Corrado et al 2010).

\section{Conclusions}

In this paper we have reported the synthesis of TPA capped $\mathrm{ZnS}: \mathrm{Cu}$ nanoparticles by simple aqueous method. Optical studies revealed that the nanoparticles formed were monodispersed (DPI 1). The PL spectrum shows a broad emission peak at around $460 \mathrm{~nm}$. Doping $\mathrm{Cu}^{2+}$ in the $\mathrm{ZnS}$ nanoparticles causes a red-shift in the emission peak.

\section{Acknowledgement}

The authors are thankful to Dr A L S Chandel, Department of Chemistry, Govt. E R Rao Science PG College, Bilaspur, for providing experimental facilities. 


\section{References}

Brus L E 1986 J. Phys. Chem. 902555

Chan W C W and Nie S 1998 Science 2812016

Corrado C, Hawker M, Livingston G, Medling S, Bridges F and Zhang J Z 2010 Nanoscale 21213

Crisponi D et al 2002 Polyhedron 211319

Daniel M C and Astruc D 2004 Chem. Rev. 104293

Ehlert O, Bucking W, Riegler J, Merculov A and Nann T 2008 Microchim. Acta 16035

Guinier A 1963 X-ray diffraction (San Francisco: W H Freeman)

Havanscak K 2003 Mater. Sci. Forum 415-416 85

Jun Y W, Jang J T and Cheon J W 2006 Bull. Korean Chem. Soc. 27961

Kuzuya T, Tai Y, Yamamuro S and Sumiyama K 2005 Sci. Technol. Adv. Mater. 684
Larson D R et al 2003 Science 3001434

Manzoor K, Vadera S R, Kumar N and Kutty T R N 2004 Solid State Commun. 129469

Moffit M, McMahon L, Pessel V and Eisenberg A 1995 Chem. Mater. 71185

Panda S K and Chaudhuri S 2007 J. Coll. Interf. Sci. 313 338

Pradeep T 2007 Nano: the essentials (USA: Tata Mc Graw Hill) p. 1

Sapra S, Prakash A, Ghangrekar A, Periasamy N and Sarma D D 2005 J. Phys. Chem. B109 1663

Suyver J F, Wuister S F, Kelly J J and Meijernik A 2001 Nano. Lett. 1429

Warad H C, Ghosh S C, Hemtanon B, Thanachayanont C and Dutta J 2005 Sci. Technol. Adv. Mater. 6296

Zhang Y and Li Y D 2004 J. Phys. Chem. B108 17805 